\title{
NEFROCALCINOSIS Y ACIDOSIS TUBULAR RENAL PROXIMAL EN SÍNDROME DE SJÖGREN.
}

\author{
NEPHROCALCINOSIS AND PROXIMAL TUBULOPATHY IN SJÖGREN'S SYNDROME.
}

\section{Diego Federico Baenas ${ }^{1,3}$, Janet Flores Balverdi ${ }^{1}$, Soledad Retamozo ${ }^{1}$, Nadia Claudine Riscanevo ${ }^{1}$, Ana} Cecilia Álvarez ${ }^{1}$, Verónica Saurit ${ }^{1}$, Jorge Luis De la Fuente ${ }^{2}$, Francisco Caeiro'.

\section{Resumen:}

El síndrome de Sjögren primario es una enfermedad autoinmune sistémica de evolución crónica. Puede presentar compromiso renal hasta en un 30\% de los pacientes. La incidencia de tubulopatías varía de 2.6 a $33 \%$. Se manifiestan por defectos en la concentración de la orina y alteraciones hidroelectrolíticas, principalmente acidosis tubular distal y de manera excepcional acidosis tubular proximal. Estos trastornos pueden asociarse a Nefrocalcinosis y litiasis renal.

Reportamos el caso de una paciente con Sjögren primario que presentó acidosis tubular renal proximal asociada a cólicos renales recurrentes por litiasis renal y nefrocalcinosis.

Destacamos la importancia de diagnosticar acidosis tubular renal en pacientes con síndrome de Sjögren que presenten alteraciones en el sedimento urinario y desórdenes electrolíticos para evitar la nefrocalcinosis medular y las nefrolitiasis asociadas. La terapia para la corrección de la acidosis tiene como objetivo evitar la progresión del trastorno y preservar la función renal.

Palabras clave: nefrocalcinosis; acidosis tubular renal; síndrome de Sjögren primario.

\section{Abstract:}

Primary Sjögren's syndrome is a systemic and chronic autoimmune disease. Renal involvement may occur in up to $30 \%$ of patients. The incidence of tubulopathies ranges from 2.6 to $33 \%$. They are manifested by defects in the urine concentration and hydroelectrolyte alterations, mainly distal tubular acidosis and exceptionally proximal tubular acidosis. These disorders can be associated with nephrocalcinosis and renal lithiasis.

We report the case of a patient with primary Sjögren who presented proximal renal tubular acidosis associated with recurrent renal colic due to renal lithiasis and nephrocalcinosis.

We highlight the importance of diagnosing renal tubular acidosis in patients with Sjögren's syndrome that present alterations in urinary sediment and electrolyte disorders to avoid nephrocalcinosis and nephrolithiasis. Acidosis correction treatment aims to prevent the progression of the disorder and preserve renal function.

Keywords: nephrocalcinosis; renal tubular acidosis; primary Sjögren’s syndrome.

\footnotetext{
1 Servicio de Reumatología. Hospital Privado Universitario de Córdoba, Córdoba, Argentina

2 Servicio de Nefrología. Hospital Privado Universitario de Córdoba, Córdoba, Argentina

3 Email de contacto: baenashospitalprivado@gmail.com
} 


\section{Introducción}

El síndrome de Sjögren primario (SSp) es una enfermedad autoinmune sistémica, de evolución crónica ${ }^{1}$. Se caracteriza por activación de células B policlonales e infiltración linfocítica de las glándulas exocrinas, provocando sequedad de mucosas, principalmente oral (xerostomía) y ocular (xeroftalmía) ${ }^{1,2}$. Puede asociarse a manifestaciones extraglandulares en un 50-60\% de los pacientes, incluyendo afectación del estado general, cutánea, articular, pulmonar, cardiovascular, nerviosa, hematológica y nefrourológica, entre otros órganos y sistemas ${ }^{1,2}$. El compromiso renal puede ser desencadenado por infiltración linfocitaria del epitelio tubular, que puede provocar nefritis túbulointersticial (NTI), y daño glomerular por inmunocomplejos y/o crioglobulinas que pueden causar glomerulonefritis (en orden de frecuencia, glomerulonefritis membranoproliferativa crioglobulinémica, glomeruloesclerosis segmentaria focal, glomerulonefritis proliferativa mesangial, nefropatía membranosa y cambios mínimos) $)^{3,4}$. Hasta un $35 \%$ de los pacientes con epitelitis linfocítica tubulointersticial pueden presentar alteración en la capacidad de acidificación de la orina ${ }^{3-}$ ${ }^{5}$. Este trastorno se manifiesta clínica y funcionalmente como acidosis tubular distal o tipo I (ATRd) en la mayoría de los casos, aunque se ha reportado infrecuentemente asociación con acidosis tubular proximal o tipo II (ATRp) con síndrome de Fanconi³.

\section{Métodos}

Presentamos un caso de SSp asociado a ATR proximal, litiasis renal y nefrocalcinosis. Se respetaron los principios de la Declaración de Helsinki.

\section{Reporte de caso}

Mujer de 62 años, con SSp de 30 años de evolución, caracterizado por xerostomía, xeroftalmía, poliartritis simétrica, test de Schirmer positivo, anticuerpos antinucleares (Hep2) 1/160 patrón moteado fino, antiRo/SSA y anti-La/SSB positivos, biopsia de glándulas salivales con sialoadenitis grado 4 (Chisholm y Mason) y ESSDAI ${ }^{2}$ (EULAR Sjögren's Syndrome Disease Activity Index) 3 al diagnóstico. Serologías virales (hepatitis B y C, HIV negativas), complemento normal, factor reumatoideo, antiDNA negativos. El proteinograma por electroforesis evidenció hipergammaglobilinemia policlonal, con inmunofijación en sangre y orina negativas. No se detectaron crioglobulinas a lo largo de su evolución. Fue tratada con hidroxicloroquina $200 \mathrm{mg} /$ día, prednisona $5 \mathrm{mg} /$ día, metotrexato $15 \mathrm{mg} / \mathrm{semana}$ y ácido fólico. En control clínico se constató proteinuria, con relación proteína/creatinina 0.8 , y sedimento urinario con leucocituria, sin hematuria. Los niveles séricos de creatinina y urea fueron normales. En su evolución presentó cólicos renales recurrentes y acidosis metabólica con anión GAP normal en algunas oportunidades. Se solicitó ecografía de aparato urinario que evidencia imágenes ecogénicas bilaterales en pirámides renales y numerosas calcificaciones litiásicas pequeñas bilaterales en grupos calicilares (figura 1.A). Estos hallazgos también se constataron en radiografía simple (figura 1.B) y en la tomografía axial computada (TAC), junto a calcificaciones papilares renales, bilaterales, sin dilatación de vías excretoras (figura 1. C y D). Se solicitó orina de 24 horas con pH de 7, densidad 1005, volumen $3500 \mathrm{ml}$, calciuria $350 \mathrm{mg}$ (valor normal 51 - 299), osmolaridad 219 mosm/l, ácido úrico 383 mg, fosfaturia 537mg (VN 600-1400), excreción normal de oxalato y $53 \mathrm{mg}$ de citrato (300-900). Presentó glucosuria con glucemia normal. En algunas consultas se evidenció aumento de excreción urinaria de potasio. Se descartó gammapatía monoclonal. Los estudios de metabolismo fosfo-cálcico revelaron hipocalcemia $7.5 \mathrm{mg} / \mathrm{dl}$, hipofosfatemia $2.2 \mathrm{mg} / \mathrm{dl}$, paratohormona 45 $\mathrm{pg} / \mathrm{ml}$. El ESSDAl fue 6 al momento del compromiso renal. El hallazgo de proteinuria en todos los estudios fue de origen tubular. 


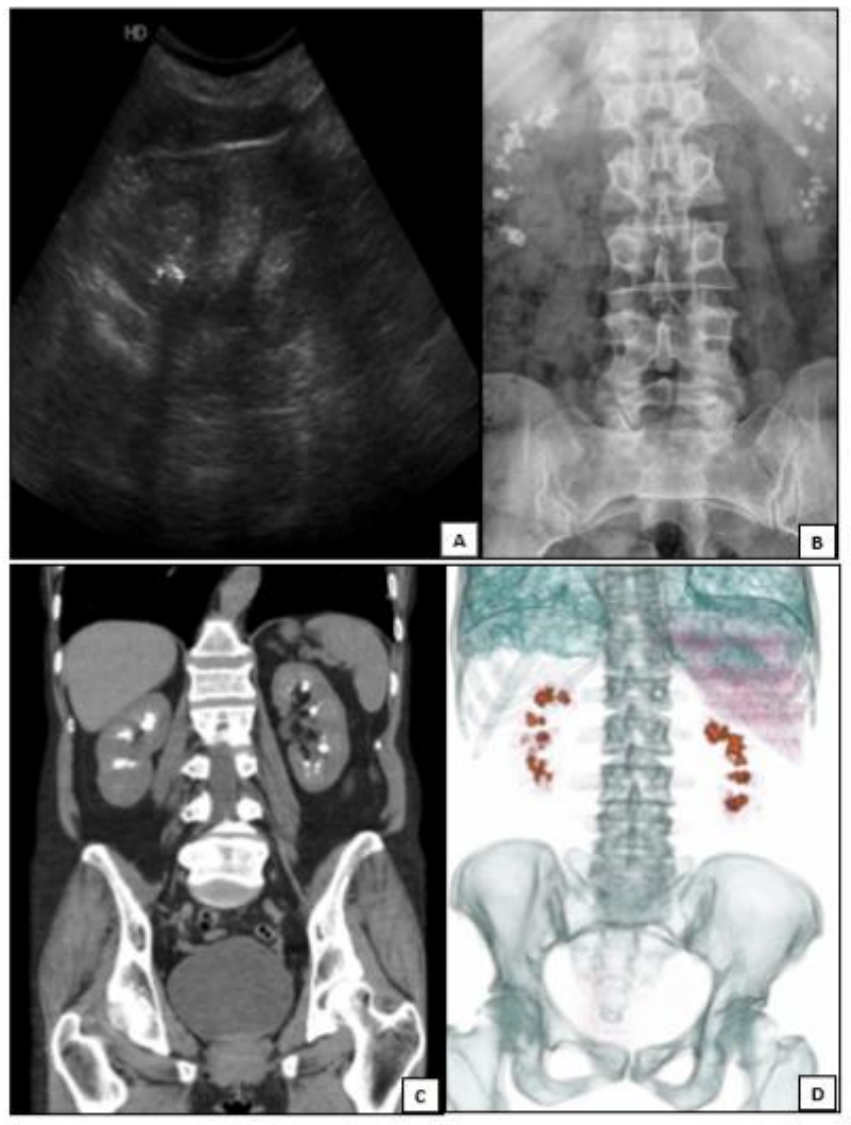

Figura 1A, Figura 1B, Figura 1C y Figura 1D

Se diagnosticó nefrocalcinosis en contexto de SSp con acidosis tubular renal (ATR) y parámetros de tubulopatía proximal. Se agregó citrato de potasio al tratamiento, con buena respuesta.

\section{Discusión}

EI SSp puede presentar compromiso renal con una frecuencia muy variable, según distintas series, que oscila entre el 2 a $>30 \%^{1-3}$. Suele observarse en pacientes mayores de 50 años y luego de 2 a 7 años del diagnóstico de $\mathrm{SSp}^{3}$. La NTI es la afectación más común entre 54-80\%, con una evolución aguda o crónica ${ }^{1-}$ 5 . Suele tener una presentación insidiosa, con discretas alteraciones hidroelectrolíticas al inicio, modesta elevación de la creatinina, y proteinuria de bajo rango ${ }^{3}$. Debido a que el trastorno es tubular, a menudo los métodos convencionales (que miden albuminuria) pueden arrojar falsos negativos. La incidencia de tubulopatías varía de 2.6 a $33 \% \%^{3,6,7}$. Puede afectar cualquier segmento de la nefrona, generando defectos en la concentración de la orina y alteraciones hidroelectrolíticas plasmáticas y urinarias entre las que se destacan: ATR tipo I (la más frecuente), ATR tipo II, síndrome de Gitelman adquirido y síndrome de Bartter adquirido ${ }^{3-7}$

La tubulopatía distal (ATR I) suele provocar hipostenuria, hipercloremia e hipopotasemia ${ }^{8,9}$. Puede ser un hallazgo incidental o manifestarse por síntomas leves en la mayoría de los $\operatorname{casos}^{8}$. Sin embargo, se han reportado complicaciones más significativas como litiasis renal y nefrocalcinosis que derivan en deterioro de la función renal, y casos severos de parálisis hipocalémica ${ }^{3,10-12}$. La afectación tubular crónica puede provocar diabetes insípida nefrogénica secundaria ${ }^{4}$.

En nuestra paciente se constataron alteraciones sugestivas de compromiso tubular proximal. La ATR tipo II y el síndrome de Fanconi son excepcionales ${ }^{9}$, y afectan sólo un 3-4\% de los pacientes con SSp ${ }^{3}$. Debe sospecharse cuando existe acidosis con anión gap normal, niveles séricos disminuidos de fósforo y ácido úrico y/o glucosuria con glucemia normal ${ }^{3,4-8}$. Debe interrogarse sobre exposición a tóxicos (plomo, mercurio) y fármacos (ifosfamida -asociada a cistitis intersticial-, tenofovir) ${ }^{3}$. A pesar de que la paciente tenía diagnóstico constatado de SSp, es mandatorio descartar gammapatías monoclonales (ATR asociada a proteína de Bence-Jones) $)^{3-6}$.

Para detectar NTI y disfunción tubular, se sugiere solicitar proteinuria tubular, creatinina sérica, electrolitos plasmáticos y urinarios, $\mathrm{pH}$ urinario matutino y osmolaridad ${ }^{3}$. Habitualmente tiene buen pronóstico, pero puede generar insuficiencia renal crónica de origen parenquimatoso o posrenal (por litiasis renal y/o 
nefrocalcinosis) e hipertensión arterial secundaria ${ }^{3-7}$. Ante un paciente con SSp y alteraciones hidroelectrolíticas séricas y urinarias, debe sospecharse la posibilidad de NTI, y valorar su confirmación a través de biopsia renal ${ }^{3}$.

Tanto la ATR tipo I como la tipo II pueden causar nefrocalcinosis debido a hipercalciuria y acidosis ${ }^{10-12}$. Este proceso suele ser identificable por radiografía de abdomen con una sensibilidad (S) de $63-66 \%{ }^{12}$. La ecografía (S 85-90\%) también es un excelente método de detección temprana, y demostró ser superior a la TAC (S 81-86\%) en el manejo clínico y la monitorización de los pacientes con nefrocalcinosis leve a moderada ${ }^{13,14}$. Por estos motivos, y debido a la desventaja de la accesibilidad y efectos adversos de la radiación, la pieloTAC se reserva para casos severos, dudas diagnósticas y para intentar evidenciar si existe litiasis renal asociada ${ }^{13}, 14$. La resonancia magnética presenta mala visualización por lo que se desaconseja ${ }^{14}$.

No existe consenso respecto al tratamiento de la NIT ${ }^{3-8}$. En general se acepta el uso de esteroides cuando hay deterioro de la función renal y proteinuria significativa, asociando inmunosupresores en casos severos o cuando se requieran dosis elevadas de corticoides ${ }^{3-9}$. Las alteraciones electrolíticas con función renal conservada se pueden tratar con suplementos orales con bicarbonato y/o citrato de potasio ${ }^{3-9}$. Cuando se asocian a tubulitis, el control de la NTI con esteroides suele ser suficiente ${ }^{3,4}$.

Destacamos la importancia de sospechar NTI e investigar nefrocalcinosis medular y/o nefrolitiasis asociada a ATR en pacientes con SS con alteraciones del sedimento urinario, proteinuria e historia de cólicos renales. La terapia para la corrección de la acidosis tiene como objetivo evitar la progresión del trastorno y preservar la función renal ${ }^{3-9}$.

\section{Bibliografía}

1. Brito-Zerón P, Baldini C, Bootsma H, et al. Sjögren syndrome. Nat Rev Dis Primers. 2016 Jul 7;2:16047.

2. Ramos-Casals $M$, Brito-Zerón $P$, Seror $R$, et al. Characterization of systemic disease in primary Sjögren's syndrome: EULAR-SS Task Force recommendations for articular, cutaneous, pulmonary and renal involvements. Rheumatology. 2015;54(12):2230-2238.

3. François H, Mariette X. Renal involvement in primary Sjögren syndrome.Nat Rev Nephrol. 2016 Feb;12(2):82-93.

4. Evans R, Zdebik A, Ciurtin C, Walsh SB. Renal involvement in primary Sjögren's syndrome. Rheumatology (Oxford). 2015; 54:1541-8.

5. Goules AV,Tatouli IP, Moutsopoulos, HM, Tzioufas AG. Clinically significant renal involvement in primary Sjögren's syndrome: clinical presentation and outcome. Arthritis Rheum. 65, 2945-2953 (2013).

6. Evans RDR, Laing CM, Ciurtin C, Walsh SB. Tubulointerstitial nephritis in primary Sjögren syndrome: clinical manifestations and response to treatment. BMC Musculoskeletal Disorders. 2016;5(17):p. 2.

7. Maripuri S, Grande JP, Osborn TG, et al. Renal involvement in primary sjögren's syndrome: a clinicopathologic study. Clinical Journal of the American Society of Nephrology. 2009;4(9):1423-1431.

8. Bossini N, Savoldi S, Franceschini F, et al. Clinical and morphological features of kidney involvement in primary Sjögren's syndrome.Nephrol Dial Transplant. 2001;16(12):2328.

9. Haque SK, Ariceta G, Batlle D. Proximal renal tubular acidosis: a not so rare disorder of multiple etiologies. Nephrol. Dial. Transplant. 27, 4273-4287 (2012).

10. Polanco NA, Soto-Abraham MV, Rodríguez-Castellanos FE. Nephrocalcinosis and distal renal tubular acidosis in Sjögren's syndrome. Nefrologia 2013;33:860-1.

11. Rajput $R$, Sehgal $A$, Jain $D$, Sen $R$, Saini $O$. Nephrocalcinosis: a rare presenting manifestation of primary Sjögren's syndrome. Mod Rheumatol. 2012 Jun;22(3):479-82.

12. Gharbi C, Rottembourg J, and Izzedine H. Nephrocalcinosis in primary Sjögren's syndrome. Clin Kidney J. 2014 Apr; 7(2): 217.

13. Cheidde L, Ajzen SA, Tamer Langen CH, Christophalo D, Heilberg IP. A critical appraisal of the radiological evaluation of nephrocalcinosis. Nephron Clin Pract. 2007;106(3):c119.

14. Boyce AM, Shawker TH, Hill SC, et al. Ultrasound is superior to computed tomography for assessment of medullary nephrocalcinosis in hypoparathyroidism. The Journal of clinical endocrinology and metabolism. 2013;98:989-94. 